\title{
Respostas Cardiorrespiratórias Durante Exercício em Portadores de Transplante Cardíaco. Análise Ergoespirométrica Comparativa com Indivíduos Normais
}

A na Fátima Salles, Japy Angelini O liveira Fon, Turíbio Leite de Barros N eto, Dirceu Rodrigues de Almeida, Antonio Carlos de Camargo Carvalho, Yara Juliano, Ênio Buffolo, Eulógio Emílio Martinez Fo

São Paulo, SP

Objetivo - Avaliar as respostas cardiorrespiratórias dos portadores de transplante cardíaco (TxC).

Métodos - Submeteram-se a testes ergoespirométricos 9 portadores de TxC(GI), pareados por sexo, idade, peso e altura, com 9 indivíduos sedentários, aparentemente sadios (GII). Os pacientes eram do sexo masculino,

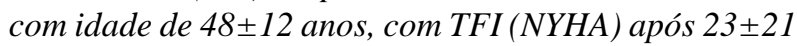
meses TxC. Faziam uso regular de ciclosporina, azatioprina, prednisona, dipiridamol e anti-hipertensivos. Os testes foram limitados por sintomas e interrompidos por exaustão.

Resultados - No pico do exercício, o GI apresentou desempenho significativamente inferior ao GII quanto ao $\mathrm{VO}_{2}, \mathrm{VE}, \mathrm{VEO}_{2}, \mathrm{FC}$, tempo de endurance e potência. No limiar anaeróbio, o GI apresentou $\mathrm{VO}_{2}$, tempo de endurance e potência significativamente inferior a do GII. Na potência de 40W o desempenho dos dois grupos foi similar.

Conclusão - O GI apresentou desempenho cardiorrespiratório significativamente inferior no pico do exercício e similar na potência de $40 \mathrm{~W}$ em relação ao GII, evidenciando os benefícios do TxC para cardiopatas em atividades habituais

Palavras-chave: transplante cardíaco, ergoespirometria, exercício físico

\section{Cardiorespiratory Response During Exercise in Heart Transplant Recipients Comparated to Normal Healthy Subjects}

Purpose - To evaluate the cardiorespiratory response of heart transplant $(H T)$ recipients.

Methods - Nine HT recipients (GI) underwent ergospirometric tests and were compared to 9 apparently healthy, sedentary subjects with similar sex, age, weight and height (GII). All were male patients aging $48 \pm 12$ years, in functional class I (NYHA) an average of $23 \pm 21$ months after HT. They were receiving cyclosporin, azathioprine, prednisone, dipyridamole and antihypertensive drugs. The tests were symptom-limited and they were interrupted due to exhaustion.

Results - During peak exercise, GI had a significantly lower physical performance related to lower $V O_{2}, V E$, $V E O_{2}, H R$, endurance time and work load. At the anaerobic threshold, $\mathrm{VO}_{2}$, endurance time and work load levels were also significantly lower in GI. The physical performance was similar between the groups in the 40W load.

Conclusion - The cardiorespiratory performance in GI was significantly lower at peak exercise and similar to GII in the 40W load, showing the HT benefits cardiac patients during usual activities.

Key-words: heart transplant, ergoespirometry, physical performance

Arq Bras Cardiol, volume $70\left(n^{0} 1\right), 15-18,1998$

O transplante cardíaco $(\mathrm{TxC})$ tornou-se o tratamento corrente para os indivíduos com insuficiência cardíaca terminal, elevando a sobrevida para mais de $80 \%$ no $1^{\circ}$ ano ${ }^{1}$ ea

Escola Paulista de Medicina - UNIFESP

Correspondência: Ana Fátima Salles - Escola Paulista de Medicina - UNIFESP Rua Botucatu, 740 - 04023-062 - São Paulo, SP

Recebido para publicação em 7/8/97

Aceito em 16/10/97 mais de $50 \%$ em 10 anos $^{2}$. Além disso, trouxe uma melhora muitas vezes considerável na qualidade de vida permitindo o retorno às atividades laborativas ${ }^{3}$.

A ergoespirometria tem sido utilizada, em portadores de TxC, para o estudo das variáveis cardiocirculatórias, hemodinâmicas e metabólicas. Comparando-se pacientes transplantados a controles aparentemente sadios, tem-se descrito níveis inferiores da capacidade aeróbia, freqüência 
cardíaca (FC) e ventilação pulmonar no pico do exercício ${ }^{4-16}$. Registram-se valores mais elevados da resistência vascular sistêmica, pressão capilar pulmonar, pressão de átrio direito e reduções do volume sistólico às custas de disfunção diastólica ${ }^{4-16}$.

O presente estudo tem por objetivo avaliar as respostas cardiorrespiratórias dos portadores de TxC ortotópico, principalmente em níveis de exercício abaixo do limiar anaeróbio (LA), onde ocorrem, geralmente, as atividades físicas habituais.

\section{Métodos}

Estudaram-se nove pacientes com TxC ortotópico (grupo I), do sexo masculino, idade de $48 \pm 12$ anos, após $23 \pm 21$ meses de cirurgia, entre dezembro/91 a janeiro/92. Os pacientes estavam em TFI (NYHA), tendo sido transplantados por cardiomiopatia dilatada (sete) e cardiopatia isquêmica (dois); faziam uso regular de ciclosporina, azatioprina, prednisona, dipiridamol e anti-hipertensivos diversos. Seis pacientes tiveram rejeição; o intervalo de tempo entre o episódio de rejeição e o teste ergoespirométrico (TEE) foi de um a 41 meses. Utilizaram-se nove voluntários, aparentemente sadios ${ }^{17}$, sem atividade física regular, pareados por sexo, idade, peso e altura, como grupo controle (grupo II).

TEE foi realizado em temperatura de $19 \mathrm{a} 24^{\circ} \mathrm{Ce}$ umidade relativa do ar de $55 \%{ }^{18}$, na vigência das medicações usuais. Utilizou-se protocolo de rampa com incrementos de $5 \mathrm{~W} / \mathrm{min}$, após estágio inicial de $3 \mathrm{~min}$ em $25 \mathrm{~W}$ a $50 \mathrm{rpm}$. TEE foi sintoma limitante, sendo interrompido por exaustão em todos os casos. Aferiram-se a FC por sensor no lóbulo da orelha e a pressão arterial (PA) por esfigmomanômetro de mercúrio. Registrou-se o eletrocardiograma em derivação $\mathrm{CM}_{5}$. O consumo de oxigênio $\left(\mathrm{VO}_{2}\right)$ foi medido a cada minuto em circuito aberto através de válvula respiratória de baixa resistência (Hans Rudolf), pela leitura das frações expiradas de $\mathrm{O}_{2}$ e $\mathrm{CO}_{2}$ em sistema metabólico (Vacumed - Applyed Electhrochemistry). A ventilação pulmonar (VE) foi obtida em ventilômetro mecânico (Singer - American Meter Division). Utilizou-se cicloergômetro eletromagnético (Caloi - Cateye Ergociser EC-1500). Denominou-se grau de deficiência aeróbia à diferença percentual entre $\mathrm{o} \mathrm{VO}_{2}$ de cada paciente do GI em relação ao seu par do GII:

$$
\mathrm{GDA} \%=\frac{\mathrm{VO}_{2} \mathrm{GII}-\mathrm{VO}_{2} \mathrm{GI}}{\mathrm{VO}_{2} \mathrm{GII}}
$$

A cinética da $\mathrm{FC}$ foi dada pela fórmula:

$$
\Delta \mathrm{FC}-\mathrm{FC}_{\mathrm{LA}}-\mathrm{FC}_{25 \mathrm{~W}}
$$

$\Delta$ WR - "carga de trabalho" $x$ tempo onde: carga de trabalho é o aumento da potência por minuto $(5 \mathrm{~W} / \mathrm{min})$

Tempo é o intervalo de tempo compreendido entre o final do $3^{\circ} \mathrm{min}$ de exercício na potência de $25 \mathrm{~W}$ e o LA.

A análise estatística constou do teste de MannWhitney para comparar os grupos I e II; do coeficiente de correlação de Spearman para correlacionar o tempo de

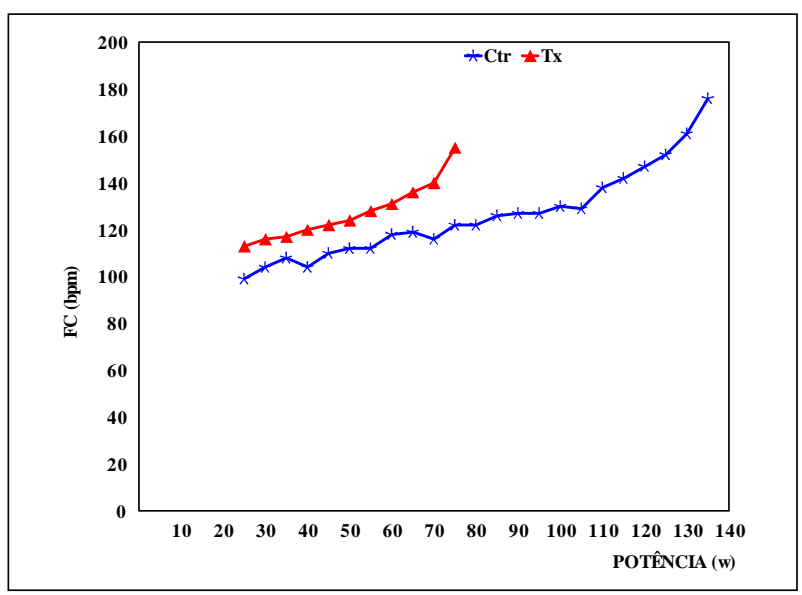

Fig. 1 - Curva de frequiência cardíaca $(\mathrm{FC})$ em bpm versus potência em $w$ no paciente $\mathrm{n}^{\circ}$ 7 em relação ao seu controle normal.

transplante com as variáveis cardiorrespiratórias e a cinética daFC com a incidência de rejeição. Fixou-se em 0,05 ou 5\% ( $<<0,05)$ o nível de rejeição de hipótese de nulidade assinalando-se com um asterisco $(*)$ os valores significantes.

\section{Resultados}

Os resultados demonstraram que o desempenho dos pacientes com TxC (GI) no pico do exercício foi inferior ao dos indivíduos controles (GII), constatando-se diferenças significantes na média de $\mathrm{VO}_{2}, \mathrm{VE}$, equivalente ventilatório para o oxigênio $\left(\mathrm{VEO}_{2}\right), \mathrm{FC}$, tempo de endurance e potência. O grau de deficiência aeróbia foi de $32 \%$ (tab. I).

No limiar anaeróbio, o GI apresentou média de $\mathrm{VO}_{2}$, tempo de endurance e potência significativamente inferior a do GII; o grau de deficiência aeróbia alcançou 26\% (tab.II). Na potência de $40 \mathrm{~W}$ os valores obtidos de $\mathrm{VO}_{2}, \mathrm{VE}, \mathrm{VEO}_{2}$, $\mathrm{VECO}_{2} \mathrm{eFC}$ não mostraram diferenças significativas entre os grupos I e II (tab. III). Não houve correlação entre o tempo de transplante (T.Tx) e o $\mathrm{VO}_{2}$ ou GDA\% no pico do exercício, no LA e na potência de 40W (tab. IV). A cinética da FC

\begin{tabular}{|lcc|}
\hline \multicolumn{2}{|c|}{ Tabela I - Variáveis cardiorrespiratórias na potência pico expressas } \\
em média
\end{tabular}




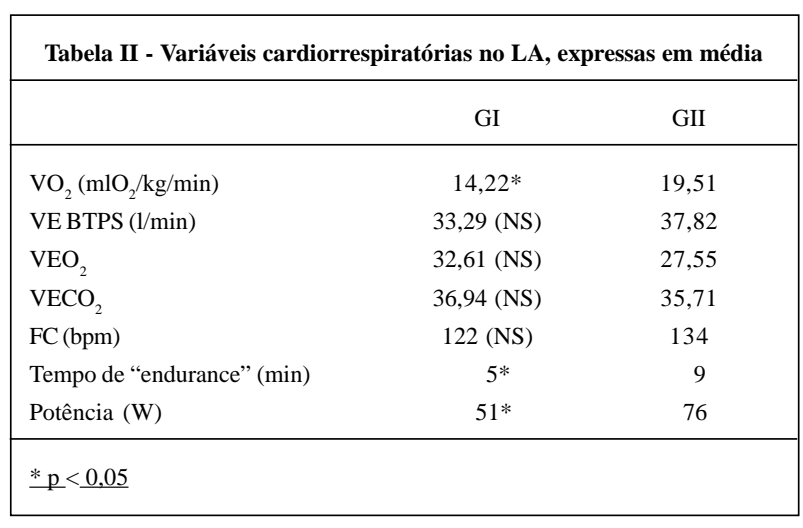

\begin{tabular}{|lcc|}
\hline \multicolumn{3}{|c|}{ Tabela III - Variáveis cardiorrespiratórias na potência de 40W, } \\
expressas em média
\end{tabular}

\begin{tabular}{|c|c|c|}
\hline \multicolumn{3}{|c|}{$\begin{array}{c}\text { Tabela IV - Correlações entre o tempo de transplante e } \mathrm{VO}_{2} \text {; e entre o } \\
\text { tempo de transplante e grau de deficiência aeróbia em diferentes } \\
\text { níveis de exercício }\end{array}$} \\
\hline Correlações & $r_{\text {calculado }}$ & $\mathrm{r}_{\text {crítico }}$ \\
\hline T. Tx $x \mathrm{VO}_{2}$ pico & $-0,31$ & 0,67 \\
\hline T. Tx $x$ LA & $-0,18$ & 0,67 \\
\hline T. Tx $x \mathrm{VO}_{2} 40 \mathrm{~W}$ & $-0,15$ & 0,66 \\
\hline T. Tx $x$ GDA $\%$ pico & $+0,23$ & 0,60 \\
\hline T. Tx $x$ GDA $\%$ LA & $-0,25$ & 0,60 \\
\hline T. Tx $x$ GDA $\% 40 \mathrm{~W}$ & $-0,16$ & 0,66 \\
\hline \multicolumn{3}{|l|}{$\underline{r}_{\text {calculado- }}>\underline{r}_{\text {critico }}$} \\
\hline $\begin{array}{l}\text { T.Tx- tempo de transpla } \\
\text { tagem; r- coeficiente de }\end{array}$ & $\begin{array}{l}\text { e defici } \\
\text { man. }\end{array}$ & em percen- \\
\hline
\end{tabular}

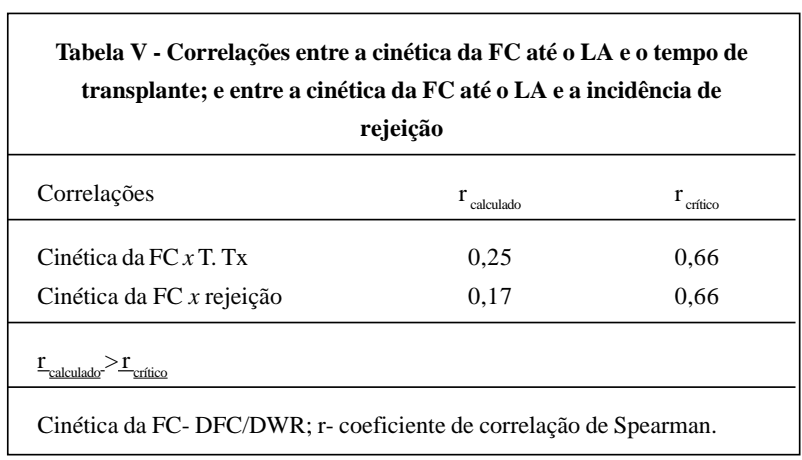

em exercício submáximo até o LA não mostrou diferenças significativas entre os grupos (fig. 1). Não houve correlação entre a cinética da FC até oLA e o T.Tx e a cinética da FCe a incidência de episódios de rejeição (tab. V).

\section{Discussão}

Vários autores têm demonstrado redução da capacidade aeróbia máxima em portadores de $\mathrm{TxC}$, quando comparados a controles, aparentemente sadios ${ }^{4,5,7,8,10,12,13,15,19,20-22}$. Calculando-se o grau de deficiência aeróbia, em relação a indivíduos controles aparentemente sadios, classificados segundo diferentes critérios, observam-se variações entre 30 a $50 \%(44,75 \% \pm 7,85)$, no presente estudo atingindo a $32 \%$. Múltiplos fatores parecem ser responsáveis por esta redução. A incompetência cronotrópica secundária à desnervação cardíaca e à disfunção diastólica de causa não conhecida reduzem o débito cardíaco afetando o desempenho do coração transplantado ${ }^{15,16,22,23}$. Há redução da extração periférica de oxigênio ${ }^{10,15,22,24-26}$. A resposta neuroendócrina exagerada ${ }^{12} \mathrm{e}$ a redução da capacidade de difusão pulmonar ${ }^{27,28}$ parecem afetar também a tolerância ao exercício. Os baixos níveis de potência e do tempo de endurance descritos neste estudo ilustram o menor desempenho dos Tx no pico do exercício.

Têm-se relatado valores significativamente inferiores de VEno pico do exercício com equivalente respiratório para $\mathrm{CO}_{2}$ mais elevado ${ }^{13}$. O mecanismo responsável pela resposta ventilatória exagerada não está esclarecido. Há alterações da relação ventilação/perfusão pulmonares com conseqüente aumento do espaço morto fisiológico ${ }^{4,29}$, e disfunção da musculatura respiratória ${ }^{30,31}$ atribuídas à resposta atenuada do débito cardíaco durante o exercício.

O limiar anaeróbio tem variado de 49 a $63 \%$ do $\mathrm{VO}_{2}$ pico, em homens aparentemente sadios ${ }^{32-35}$. Nos transplantados ele ocorre em percentuais mais elevado do $\mathrm{VO}_{2}$ pico ${ }^{13}$. Neste estudo, o LA ocorreu em 71,44\% e 63,75\% do $\mathrm{VO}_{2}$ pico, respectivamente no GIe GII. Embora, sem significância estatística, a média dos valores do LA no GI aproximou-se mais do $\mathrm{VO}_{2}$ pico que no GII. Em valores absolutos, o LA foi significativamente inferior no GI, com GDA de $26 \%$. Resultados semelhantes foram relatados por Marzo e $\mathrm{col}^{13}$. O tempo de endurance e a potência atingidos neste nível de exercício também foram significativamente inferiores. O desempenho dos TxC no LA poderia refletir um nível de esforço próximo do pico do esforço e estaria sujeito às mesmas influências dos fatores que interferem neste nível de exercício.

A maioria das atividades físicas diárias corresponde a exercícios submáximos classificados como leves e moderados, que ocorremem níveis de até $5 \mathrm{MET}\left(17,5 \mathrm{mlO}_{2} / \mathrm{kg} / \mathrm{min}\right)^{19}$. No presente estudo, os grupos I e II atingiram, em potência de $40 \mathrm{~W}$, médias de $\mathrm{VO}_{2}$ de respectivamente, $12,34 \mathrm{e} 12,38 \mathrm{mlO}_{2} /$ $\mathrm{kg} / \mathrm{min}$, não havendo diferença significativa entres os valores (tab. III). Resultados semelhantes foram relatados na literatura. Meyer e col em cargas submáximas de $50 \mathrm{~W}$ encontraram médias de $\mathrm{VO}_{2}$ similares nos grupos controle e transplantados ${ }^{14}$. Degré e $\mathrm{col}^{36}$ realizaram estudo comparativo 
entre transplantados e pacientes previamente submetidos à cirurgia de revascularização miocárdica. Os transplantados apresentaram médias de $\mathrm{VO}_{2}$ em níveis submáximos de exercício uniformemente inferiores. Entretanto, as diferenças foram estatisticamente significantes apenas a partir de potência de $80 \mathrm{~W}^{6}$. Martin e $\mathrm{col}^{16} \mathrm{e} \mathrm{Kao} \mathrm{e} \mathrm{col}{ }^{15}$ relataram menor volume sistólico em TxC em exercício submáximo, na posição ortostática; relacionaram o achado à redução do volume diastólico final devido a disfunção diastólica ventricular. Degré e $\mathrm{col}^{36}$ encontraram, em níveis submáximos de esforço até $50 \%$ da capacidade aeróbia, valores de frequência cardíaca significativamente mais elevados. Neste estudo, os grupos I e II mostraram médias de FC de 119bpm e 114 bpm, com tendência não significativa de valores mais eleva- dos no Tx.Em Tx, a resposta mais elevada da FC em exercício submáximo tem sido atribuída a aumento dos níveis plasmáticos de catecolaminas, da densidade de betarreceptore ${ }^{37} \mathrm{e}$ a efeito cronotrópico positivo intrínseco no marcapasso induzido pelo retorno venoso ${ }^{38-40}$. Tem-se descrito, ainda, como mecanismo compensatório da resposta atenuada de débito cardíaco em Tx, em exercício submáximo, o aumento na diferença artério-venosa de oxigênio ${ }^{29}$.

Este estudo encontrou um desempenho cardiorrespiratório do grupo dos transplantados significativamente inferior no pico do exercício e similar na potência de 40W, quando comparados aos seus pares aparentemente sadios, evidenciando os benefícios do TxC para os cardiopatas durante suas atividades físicas habituais.

\section{Referências}

1. Hunt SA, Bristow MR, Kubo SH, O'Connel JB, Young JB - Task Force 8: Training in heart failure and transplantation. J Am Coll Cardiol 1995; 25: 29-31.

2. Kaye MP-The registry of the International Society for Heart and Lung Transplantation: Tenth official report - 1993. J Heart Lung Transplant 1993; 12: 541-8.

3. Parris W, Woodbury A, Thompson S et al - Returning to work after heart transplantation. J Heart Lung Transplant 1993; 12: 46-54.

4. Pope SE, Stinson EB, Daughters GT, Schroeder JS, Ingles NG, Alderman E-Exercise response of the denervated heart in long-term cardiac transplant recipients. Am J Cardiol 1980; 46: 213-8.

5. Sawin WN, Haskell WL, Schroeder JS, Stinson EB - Cardiorespiratory responses of cardiac transplant patients to graded/symptom-limited exercise. Circulation 1980; 62: 55-60.

6. Degre SGL, Niset GL, DeSmet JM, Ibraim T, Stoupel E - Cardiorespiratory response to early exercise testing after orthotopic cardiac transplantation. Am J Cardiol 1987; 60: 926-8.

7. Cerretelli P, Grassi B, Colombini A, Caru B, Marconi C- Gas exchange and metabolic transients in heart transplant recipients. Resp Physiology 1988; 74: 355-71.

8. Banner NP, Lloyd MH, Hamilton RD, Innes JA, Guz A, Yacoub MH-Cardiopulmonary response to dynamic exercise after heart and combined heart lung transplant. Br Heart J 1989; 61: 215-23.

9. Bernardi L, Salvucci F, Suardi R, Soldá PL, Caciatti A, Perlini S, Falcone C, Ricciardi L - Evidence for an intrinsic mechanism regulating heart rate variability in the transplanted and the intact heart during submaximal dynamic exercise. Cardiovasc Res 1990; 24: 969-81.

10. Jensen RL, Yanowitz FG, Crafo RO - Exercise hemodynamics and oxygen delivery measurements using rebreathing techniques in heart transplant patients. Am J Cardiol 1991; 68: 129-33.

11. Mettauer B, Lampert E, Lonsdorfer J, Levy F, Geny B, Kretz JG Get al - Cardiorespiratory and neurohumoral response to incremental maximal exercise in patients with denervated transplanted hearts. Transplant Proc 1991; 23: 1178-81.

12. Braith RW, Wood CE, Limacher MC et al - Abnormal neuroendocrine responses during exercise in heart transplant recipients. Circulation 1992; 86: 1453-63.

13. Marzo KP, Wilson JR, Manconi DM - Effects of cardiac transplantation on ventilatory response to exercise. Am J Cardiol 1992; 69: 547-53.

14. Meyer M, Cerretelli P, Cabrol C, Püper J - $\mathrm{O}_{2}$ transport during exercise after cardiac transplantation. In: Erdmann W, Burley DP, ed. - Oxygen Transport to Tissue XIV. New York: Plenum Press, 1992: 491-6.

15. Kao AC, Trigt PV, Shaeffer-McCall GS et al-Central and peripheral limitations to upright exercise in untrained cardiac transplant recipients. Circulation 1994; 89: 2605-15

16. Martin TW, Gaucher J, Pupa LE, Seaworth JF- Response to upright exercise after cardiac transplantation. Clin Cardiol 1994; 17: 292-300.

17. Schlant RG, Blomqvist CG, Brandenburg RO et al - Guidelines for exercise testing. A report of the American College of Cardiology/American Heart Association - Task Force on assessment of cardiovascular procedures (Subcommittee on exercise testing). J Am Coll Cardiol 1986; 8: 725-38.

18. Standars for Adult Exercise Testing Laboratories - American Heart Association Subcommittee on Reabilitation, Target Activity Group. Circulation 1979; 59: 421A.

19. Hidalgo R, Alegriá E, Castello R et al - Stress testing in patients on year after orthotopic cardiac transplantation. Angiology 1989; p: 650-5.

20. Stevenson LW, Sietsena K, Tillisch JH et al - Exercise capacity for survivors of cardiac transplantation or sustained medical therapy for stable heart failure. Circulation 1990; 81: 70-85.

21. Manda KJS, Donchez LJ, Mull RL, Mancini DM - Serial assessment of exercise capacity post cardiac transplantation. Circulation 1993; 88(suppl 1): I-591.

22. Kao AC, Tright PV, Shaeffer-McCall GS et al - Allograft diastolic dysfunction and chronotropic incompetence limit cardiac output response to exercise two to six years after heart transplantation. J Heart Lung Transplant 1995; 14: 11-22.

23. Paulus WJ, Brauzwaer JGF, Felice H, Kisham N, Welleus F-Deficient acceleration on the left ventricular relaxation during exercise after transplantation. Circulation 1992; 86: 1175-85.

24. Horber FF, Hoppeler H, Scheidegger JR, Grunig BE, Howard H, Frey FJ - Impact of physical training on the ultrastructure of midthigh muscle in normal subjects and in patients treated with glucorticoids. J Clin Invest 1987; 79: 1181-90.

25. Massie B, Conway M, Rajapopalan B et al - Skeletal muscle metabolism during exercise under ischemic conditions in congestive heart failure. Evidence for abnormalities unrelated to blood flow. Circulation 1988; 78: 320-6.

26. Sinoway L, Monotti J, Dallor D et al - Delayed reversal of impaired vasodilation in congestive heart failure after heart transplantation. Am J Cardiol 1988; 61: 1076-9.

27. Rawnscraft SA, Gross CR, Kubo SH et al - Pulmonary function after sucessful heart transplantation. Chest 1993; 103: 54-8.

28. Braith RW, Limacher MC, Stafles ED, Pollock ML - Blood gas dynamics on the onset of exercise in heart transplant recipients. Chest 1993; 103: 1692-8.

29. Savin WM, Schroeder JS, Haskell WL-Response of cardiac transplant recipients to static and dynamic exercise: a review. Heart Transplant 1983; 1: 72-9.

30. Killian K, Jones N - Respiratory muscle and dyspnea. Clin Chest Med 1988; 9 : 237-48.

31. Mancini DM, Ferraro N, Nazzaro D, Change B, Wilson J - Demonstration of respiratory muscle deoxygenation during exercise in patients with heart failure using near-infrared espectroscopy. J Am Coll Cardioll 1991; 18: 492-8.

32. Wasserman K, Whipp BJ, Koyal SN, Beaer WL - Anaerobic threshold and respiratory gas exchange during exercise. J Appl Physiol 1973; 35: 236-43.

33. Dawis JA, Franl MN, Whipp BJ, Wasserman K - Anaerobic threshold alterations caused by endurance training in midle - aged men. J Appl Physiol 1979; 46: 1039-46.

34. Orr GW, Green HJ, Hughson RL, Bennett GW - A computer linear regression model to determine ventilatory anaerobic threshold. J Appl Physiol 1982; 52: 1349-52.

35. Nery LE, Wasserman K, French W, Oren A, Dawis JA - Contrasting cardiovascular and respiratory responses to exercise in mitral valve and chronic obstructive pulmonary diseases. Chest 1983; 83: 446-53.

36. Degré S, Niset G, Coustry C - Rehabilitation after cardiac transplantation: 10 year follow-up physiological aspects (in press).

37. Yusuf S, Theorofoulos S, Mathoas CJ et al - Increased human sensitivity of the denervated transplanted human heart to isoprenaline both before and after b-adrenergic blockade. Circulation 1987; 75: 696-704

38. Blinks JR - Positive chronotropic effect of increasing right atrial pressure in the isolated mamalian heart. Am J Physiol 1956; 186: 299-303.

39. Shawer JA, Leon DF, Gray S, Leonard JJ, Bahnson HT - Hemodynamic observations after cardiac transplantation. N Engl J Med 1969; 281: 822-7.

40. Bexton RS, Milne JR, Cony-Pearce R,English TAH,Camm AJ-Effect of betablockade on exercise response after cardiac transplantation. Br Heart J 1983; 49: 584-8. 